\title{
Biotic Relationships: Distribution of Antibiotic Resistance Genes in Nosocomial Pathogens
}

\author{
Tejpreet Chadha* \\ Department of Biological \& Environmental Sciences, Troy University, Troy, AL 36082 \\ *Corresponding author: tchadha@troy.edu
}

Received May 22, 2014; Revised June 23, 2014; Accepted July 13, 2014

\begin{abstract}
The strong selective pressure exerted by excessive use of antibiotics in the last decades has increased the acquisition of resistance genes by horizontal gene transfer. Horizontal gene transfer contributed to the diversification of microorganisms by influencing traits such as metabolic potential of a bacterial cell, antibiotic resistance, symbiosis, fitness, and adaptation. The study of biotic relationships helped to examine how they may contribute to virulence such as transfer of toxin genes, antibiotic resistance genes. The current study examines the pattern of distribution of Ambler (molecular) classes of $\beta$-lactamases (A, B, C, D) that provides a basic understanding and an initial assessment of resistance genes that may have a different role in the natural environment in free living and symbiotic nosocomial pathogens. The distribution of the four different Ambler (molecular) classes of $\beta$-lactamases (A, B, C, and D) differs among different bacterial species. The results from absolute abundance data showed prevalence of class B $\beta$-lactamases was highest in free living and lowest in symbiotic bacterial species. Interestingly, class D $\beta$-lactamases was absent in symbiotic bacterial species. However, relative abundance class $D \beta$-lactamases was lowest for free-living bacterial species. The prevalence of class B $\beta$-lactamases based upon the total number of sequences checked predominated in pathogens that are free living when compared to other Ambler classes of $\beta$ lactamases. The study of biotic relationships helps to understand what selective or stimulatory pressures are driving the spread of antibiotic resistance genes. In the future, this may help to design effective strategies for preventing further increases in the incidence of antibiotic-resistant bacteria.
\end{abstract}

Keywords: $\beta$-lactamases, symbiotic bacteria, free living bacteria, antibiotic resistance genes

Cite This Article: Tejpreet Chadha, "Biotic Relationships: Distribution of Antibiotic Resistance Genes in Nosocomial Pathogens.” American Journal of Microbiological Research, vol. 2, no. 4 (2014): 118-121. doi: 10.12691/ajmr-2-4-3.

\section{Introduction}

In nature, bacterial species rarely exist in isolation. They form commensal and symbiotic relationships with other microbes that may promote transfer of genes via horizontal gene transfer [1]. Horizontal gene transfer facilitates transfer of genes via mobile genetic elements such as plasmids, transposons and phages that can influence the traits such as metabolic potential of a bacterial cell, antibiotic resistance, biotic relationships and adaptation [2].

The bacterial species that produce antibiotics have antibiotic resistance genes that are mainly chromosomally encoded and are responsible for various physiological functions such as self-protection, metabolism, biosynthesis and signaling [3]. The mobility of antibiotic resistance genes from the natural environment to human pathogens is due to selective pressure exerted by excessive use of antibiotics in the last decades. Thus, acquisition of antibiotic resistant genes by horizontal gene transfer is one of the important mechanisms in the subsequent development and spread of antibiotic resistance $[4,5,6,7,8]$. For instance, the $d d l$ gene from Streptomyces toyocaensis produces a glycopeptide while the partial $d d l$ gene from Amycolatopsis orientalis produces vancomycin. They encode proteins that show a high degree of homology to the vanA and $v a n B$ genes that are present in clinical isolates that are resistant to vancomycin [9]. The emrG gene (macrolide resistance) present in Bacteroides human colonic bacteria are also found in Bacillus soil bacteria. There is $98 \%$ homology of DNA sequence of emrG genes between the two species that suggest its origin in Bacillus soil bacterial species [10].

The multidrug-resistant bacteria have been discovered to contain newer $\beta$-lactamases, extended-spectrum $\beta$ lactamases (ESBLs) that are capable of breaking up penicillin, broad-spectrum cephalosporin and monobactams [11,12]. Beta ( $\beta$ ) lactamases evolved from transpeptidases, and they have a role in cell wall synthesis $[13,14,15]$. They were probably housekeeping genes and their activity against antibiotics is a secondary response to their current environments $[5,6,16]$. Interestingly, biotic relationships such as symbiosis may promote the movement of antibiotic resistance elements from antibiotic producers to commensal and pathogenic bacteria via horizontal gene transfer.

The excessive usage of antibiotics in hospitals, agriculture, aquaculture, and livestock has resulted in the 
selection of resistant bacteria in most human-impacted habitats $[17,18,19]$. As a result, there are now fewer drugs effective against such pathogens. The mortality rate is high among persons with prolonged hospitalization that are critically ill and exposed to invasive devices such as ventilators or central venous catheters [12,20-26]. The current classification for $\beta$ - lactamases includes four molecular classes A, B, C and D based on the amino acid sequence. Molecular classes A, C, and D comprises the serine $\beta$-lactamases and class $B$ contains the zincdependent metalloenzymes $[27,28]$.

The common six pathogen that cause the majority of hospital-acquired infections in the United States are Acinetobacter baumanii, Enterobacter species, Enterococcus faecium, Klebsiella pneumonia, Pseudomonas aeruginosa and Staphylococcus aureus [29]. The current study examines the natural pattern of distribution of Ambler (molecular) classes of $\beta$-lactamases $(\mathrm{A}, \mathrm{B}, \mathrm{C}$, and $\mathrm{D})$ in common free-living and symbiotic nosocomial pathogens. The study provides a basic understanding and an initial assessment of resistance genes that may help to understand the selective or stimulatory pressures that are driving the spread of antibiotic resistance genes. In the future, this may help to design effective strategies for preventing further increases in the incidence of antibiotic-resistant bacteria.

\section{Material and Methods}

\subsection{Database}

The databases used in the study are National Center for Biotechnology Information (NCBI) [http://www.ncbi.nlm.nih.gov/], the Department of Energy (DOE) and Joint Genome Institute (JGI) [http://www.jgi.doe.gov/] [30]. The bacterial species
Acinetobacter baumannii, Escherichia coli, Enterobacter cloacae, Haemophilus influenza, Klebsiella pneumonia, Pseudomonas aeruginosa, Clostridium difficile, Enterococcus faecalis, Streptococcus pneumonia and Staphylococcus aureus were evaluated for free living, symbiosis and presence of the four different Ambler classes of $\beta$-lactamase genes (A, B, C and D).

\subsection{Data Acquisition and Organization}

The data was organized on the basis of biotic relationships, free living, symbiosis and occurrence of four different molecular classes of $\beta$-lactamases. The bacterial species A. baumannii, E. coli, E. cloacae, $H$. influenza, K. pneumonia, $P$. aeruginosa, C. difficile, E. faecalis, $S$. pneumonia and $S$. aureus with positive $\beta$ lactamases status were evaluated for the presence of four different Ambler classes of $\beta$-lactamase genes (A, B, C and D).

\subsection{Data Analysis}

The absolute numbers of species with positive $\beta$ lactamase status were examined. The relative abundances of the $\beta$-lactamases were calculated to correct for differences in the total number of bacteria sequenced within a particular group. The calculation of absolute abundance was done by looking at the total number of bacteria that were shown to have $\beta$-lactamase sequences within a taxonomic group. In order to calculate the proportional abundance, total numbers of bacteria that have $\beta$-lactamase sequences were divided by the total number of bacteria within a taxonomic group. The relative abundance was further calculated by the total number of bacteria that have $\beta$-lactamase sequences divided by the total number of bacteria for each Ambler class within a taxonomic group.

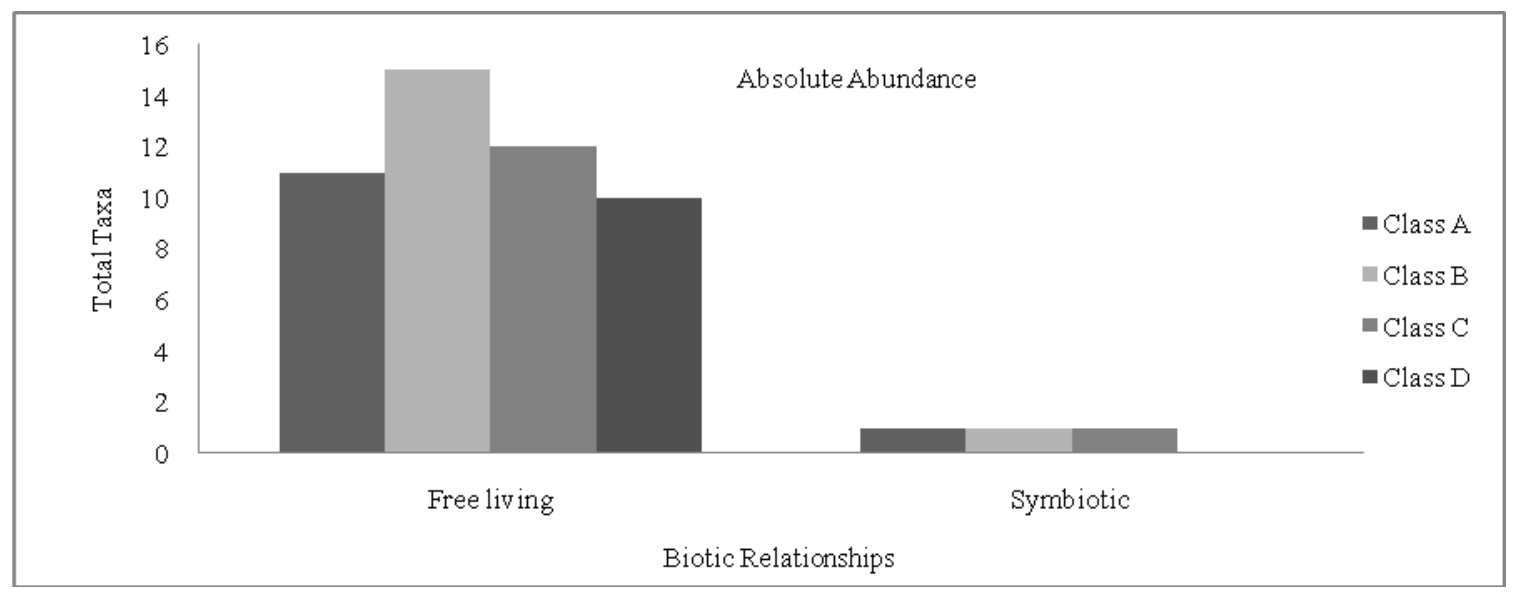

Figure 1. Distribution of the total $\beta$-lactamase occurrences by molecular class and by biotic relationships for bacterial species. Class stands for the Ambler classification of $\beta$-lactamases

\section{Results}

The data included bacterial species A. baumannii, E. coli, E. cloacae, $H$. influenza, $K$. pneumonia, $P$. aeruginosa, C. difficile, E. faecalis, S. pneumonia and $S$. aureus with positive $\beta$-lactamases status. The study examined the distributions of four different Ambler (molecular) classes of $\beta$-lactamases (A, B, C, and D) among bacterial species that are known to cause nosocomial infection. The pattern of distribution of the four different Ambler (molecular) classes of $\beta$-lactamases (A, B, C, and D) differs among different bacterial species. The absolute abundance data suggested prevalence of class B $\beta$-lactamases was highest in free living (Figure 1). The class D $\beta$-lactamases was absent in symbiotic bacterial species (Figure 1). The relative abundance of class B $\beta$-lactamases was highest when compared to 
different classes of $\beta$-lactamases within section of free living bacteria species (Figure 3). However, relative abundance class D $\beta$-lactamases was lowest for free-living bacterial species (Figure 3). Based upon the total number of sequences checked, the prevalence of class B $\beta$ lactamases predominated in pathogens that are free living when compared to other Ambler classes of $\beta$-lactamases
(Figure 1). The proportional abundance data provided evidence of the occurrence of three molecular classes (A, $\mathrm{B}$ and $\mathrm{C}$ ) of $\beta$-lactamases across free living and symbiotic bacterial pathogens (Figure 2). The proportional abundance of class D $\beta$-lactamases was highest in free living bacteria when compared to the other molecular classes of $\beta$-lactamases individually (Figure 2).

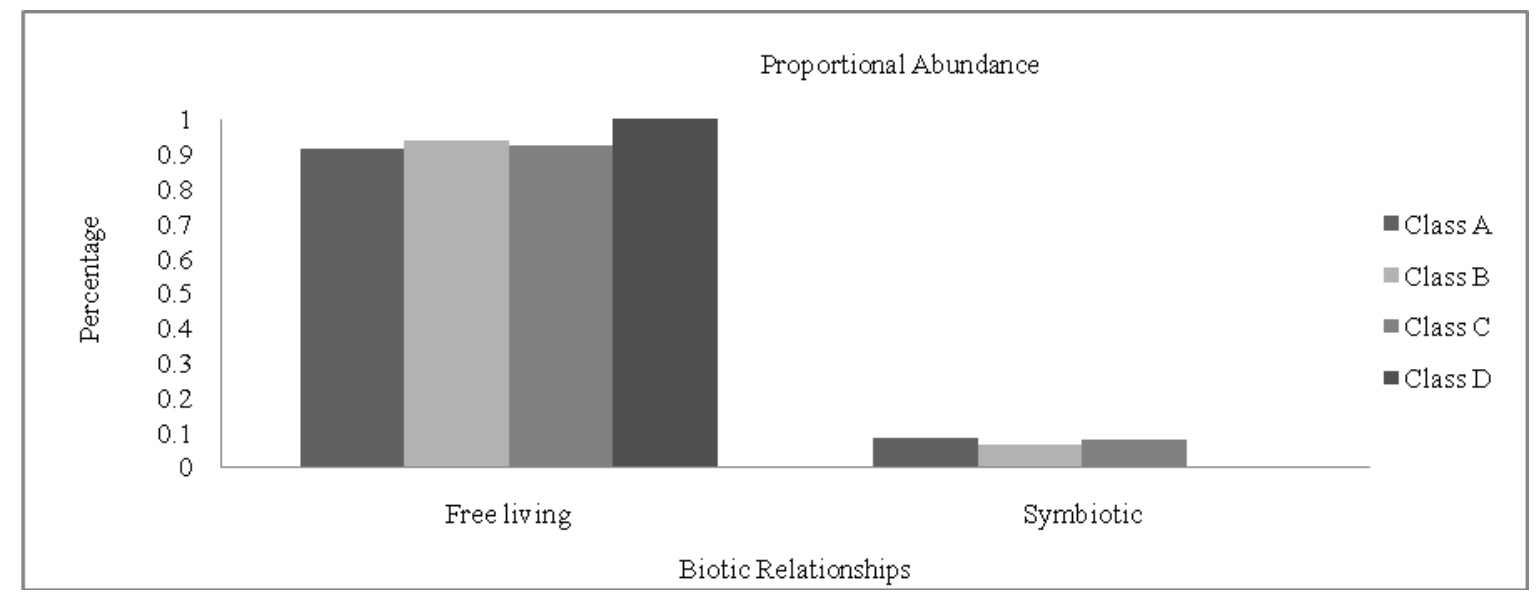

Figure 2. Proportional abundances of the total $\beta$-lactamase occurrences by molecular class and by biotic relationships for all bacterial species. Classes are defined as in Figure 1

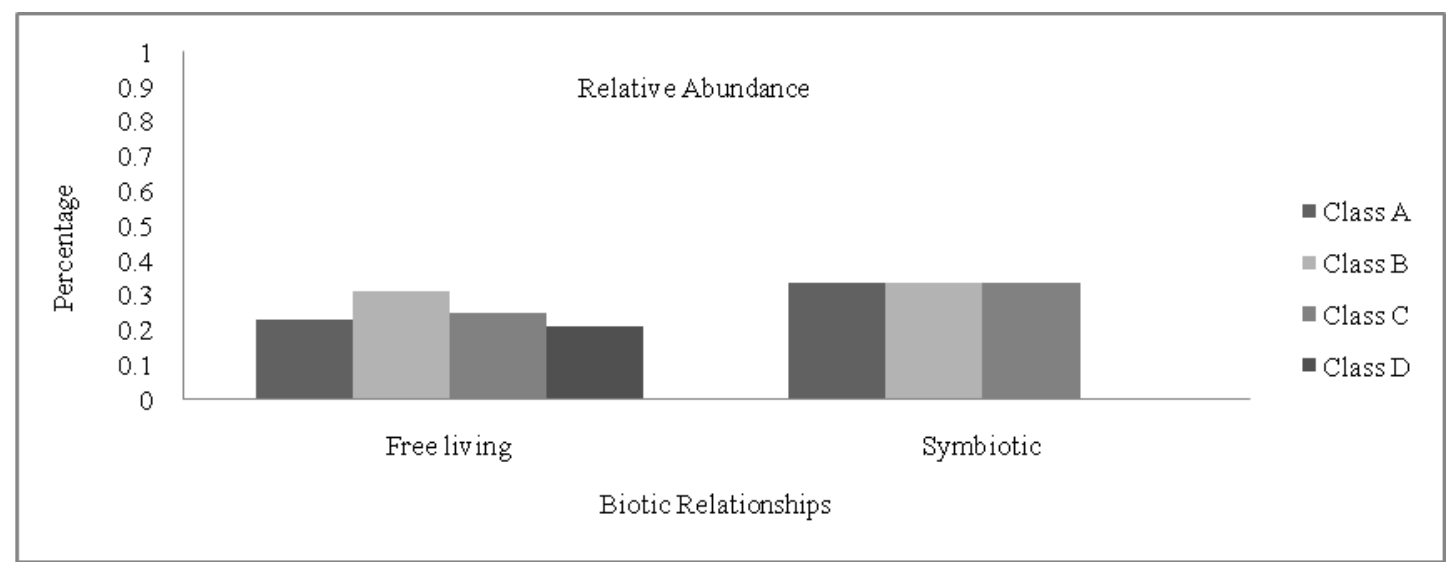

Figure 3. Relative abundances of the total $\beta$-lactamase occurrences by molecular class and by biotic relationships for all bacterial species. Classes are defined as in Figure 1

\section{Discussion}

The widespread use of $\beta$-lactam antibiotics in the treatment of human disease, animals and agriculture has resulted in the emergence of antibiotic-resistant bacterial strains globally $[6,31]$. The prevalence of antibioticresistance genes in non-antibiotic-producing bacteria may suggest that these genes probably have different physiological role as they are not under constant antibiotic selective pressure in the environment. With the high level of bacterial diversity, there is a possibility of naturally occurring bacterial species to contain a large number of antibiotic-resistance genes. The distribution pattern of $\beta$ lactamases may reflect the bacterial diversity as well as flexibility to adapt to the current changes and responses of the organisms to their environments. The evaluation of natural patterns of occurrences of $\beta$-lactamase-resistant traits will be a significant step toward understanding the distribution of $\beta$-lactamases in the environmental isolates.

The pattern of $\beta$-lactamase distributions with consideration to biotic relationships provides a basic understanding and an initial assessment of resistance genes that may have a different role in the natural environment. The data further suggest that they have indirect and direct linkages to their occurrences in the clinical environment. The study of evolution of serine $\beta$ lactamases has shown that the most prevalent molecular Ambler class are Class A $\beta$-lactamases. However, the recent research has shown that cephalosporinases (pAmpCs) are emerging a class of $\beta$-lactamases. There has been evidence that they have been moving via plasmids $[15,32,33]$. With the help of the plasmids, they move into chromosomes and spread among species and can even move across bacterial phyla. The Class D $\beta$-lactamases are a diverse group of enzymes whose genes are located both on plasmids and the chromosomes of a wide range of Gram-negative bacteria $[34,35]$. The class B metallo- $\beta$ lactamases (MBLs) have gained clinical significance because of their mobility and associated with integrons or transposons [36,37,38,39]. Interestingly, this has promoted spread of class B $\beta$-lactamase genes to other nosocomial pathogens in the hospital setting. The long history of evolution of $\beta$-lactamases also suggested the 
role in important physiological roles such as self protection that could dictate specific habitat. In this study, high occurrence of class B $\beta$-lactamases in free living bacteria may suggest that these genes could be involved in different physiological roles besides antibiotic-resistance mechanisms that appear to respond to environmental heterogeneity. The mobility of class B $\beta$-lactamase via horizontal transfer could have an effect on bacteria phenotype. However, if the selection pressure continues and once these new genes are amplified in a population, there is likely a high probability that they will be moved into clinical settings simply by random events. This data may suggest that the gain of $\beta$-lactamases genes during the course of their evolution may even contribute to their virulence.

\section{Acknowledgement}

National Center for Biotechnology Information database (http://www.ncbi.nlm.nih.gov/), Joint Genome Institute (JGI) database [http://www.jgi.doe.gov/], Department of Biological \& Environmental Sciences, Troy University. I would like to thank everyone that has helped me in this work.

\section{References}

[1] Davies, J. and D. Davies, Origins and evolution of antibiotic resistance. Microbiol Mol Biol Rev, 2010. 74(3): p. 417-33.

[2] Martinez, J.L., et al., A global view of antibiotic resistance. FEMS Microbiol Rev, 2009. 33(1): p. 44-65.

[3] Martinez, J.L., F. Baquero, and D.I. Andersson, Predicting antibiotic resistance. Nature Reviews Microbiology, 2007. 5(12): p. 958-965.

[4] Chadha, T., Antibiotic Resistant Genes in Natural Environment. Agrotechnol, 2012. 1.

[5] Allen, H.K., et al., Call of the wild: antibiotic resistance genes in natural environments. Nat Rev Microbiol, 2010. 8(4): p. 251-9.

[6] Aminov, R.I., The role of antibiotics and antibiotic resistance in nature. Environ Microbiol, 2009. 11(12): p. 2970-88.

[7] Petty, N.K., et al., Global dissemination of a multidrug resistant Escherichia coli clone. Proc Natl Acad Sci U S A, 2014. 111(15): p. 5694-9.

[8] Amos, G.C., et al., Waste water effluent contributes to the dissemination of CTX-M-15 in the natural environment. J Antimicrob Chemother, 2014. 69(7): p. 1785-91.

[9] Marshall, C.G., et al., D-Ala-D-Ala ligases from glycopeptide antibiotic-producing organisms are highly homologous to the enterococcal vancomycin-resistance ligases VanA and VanB. Proc Natl Acad Sci U S A, 1997. 94(12): p. 6480-3.

[10] Lomovskaya, O. and K. Lewis, Emr, an Escherichia coli locus for multidrug resistance. Proc Natl Acad Sci U S A, 1992. 89(19): p. 8938-42.

[11] Gupta, V., An update on newer beta-lactamases. Indian J Med Res, 2007. 126(5): p. 417-27.

[12] Pitout, J.D., et al., Emergence of Enterobacteriaceae producing extended-spectrum beta-lactamases (ESBLs) in the community. J Antimicrob Chemother, 2005. 56(1): p. 52-9.

[13] Hall, B.G. and M. Barlow, Evolution of the serine beta-lactamases: past, present and future. Drug Resist Updat, 2004. 7(2): p. 111-23.

[14] Abraham, E.P., A retrospective view of beta-lactamases. J Chemother, 1991. 3(2): p. 67-74.
[15] Meroueh, S.O., et al., Structural aspects for evolution of betalactamases from penicillin-binding proteins. J Am Chem Soc, 2003. 125(32): p. 9612-8.

[16] Martinez, J.L., The role of natural environments in the evolution of resistance traits in pathogenic bacteria. Proc Biol Sci, 2009. 276(1667): p. 2521-30.

[17] Martinez, J.L., Environmental pollution by antibiotics and by antibiotic resistance determinants. Environ Pollut, 2009. 157(11): p. 2893-902.

[18] D'Costa, V.M., et al., Sampling the antibiotic resistome. Science, 2006. 311(5759): p. 374-7.

[19] Jechalke, S., et al., Fate and effects of veterinary antibiotics in soil. Trends Microbiol, 2014.

[20] Peirano, G., et al., New Delhi Metallo-beta-Lactamase from Traveler Returning to Canada. Emerging Infectious Diseases, 2011. 17(2): p. 242-244.

[21] Lode, H., Management of serious nosocomial bacterial infections: do current therapeutic options meet the need? Clin Microbiol Infect, 2005. 11(10): p. 778-87.

[22] Ahoyo, T.A., et al., Prevalence of nosocomial infections and antiinfective therapy in Benin: results of the first nationwide survey in 2012. Antimicrob Resist Infect Control, 2014. 3: p. 17.

[23] Zhang, S., et al., Bacteriology and drug susceptibility analysis of pus from patients with severe intra-abdominal infection induced by abdominal trauma. Exp Ther Med, 2014. 7(5): p. 1427-1431.

[24] Wayenberg, L., et al., [Urinary tract infection with Escherichia coli producing extended-spectrum $\beta$-lactamase in a traveler returning from Southeast Asia]. Bull Soc Pathol Exot, 2013. 106(1): p. 1-4.

[25] Canizalez-Roman, A., et al., Prevalence and antibiotic resistance profiles of diarrheagenic Escherichia coli strains isolated from food items in northwestern Mexico. Int J Food Microbiol, 2013. 164(1): p. 36-45.

[26] Toroglu, S., H. Avan, and D. Keskin, Beta-lactamases production and antimicrobial resistance ratio of Pseudomonas aeruginosa from hospitalized patients in Kahramanmaras, Turkey. J Environ Biol, 2013. 34(4): p. 695-700.

[27] Ambler, R.P., The structure of beta-lactamases. Philos Trans R Soc Lond B Biol Sci, 1980. 289(1036): p. 321-31.

[28] Bush, K. and G.A. Jacoby, Updated functional classification of beta-lactamases. Antimicrob Agents Chemother, 2010. 54(3): p. 969-76.

[29] Rice, L.B., Federal funding for the study of antimicrobial resistance in nosocomial pathogens: no ESKAPE. J Infect Dis, 2008. 197(8): p. 1079-81.

[30] Federhen, S., The NCBI Taxonomy database. Nucleic Acids Res, 2012. 40(Database issue): p. D136-43.

[31] Jayaraman, R., Novel mechanisms of emergence of multidrug resistance/tolerance. Current Science, 2010. 99(8): p. 1008-1010.

[32] Jacoby, G.A., AmpC beta-lactamases. Clin Microbiol Rev, 2009. 22(1): p. 161-82, Table of Contents.

[33] Bauernfeind, A., Y. Chong, and K. Lee, Plasmid-encoded AmpC beta-lactamases: how far have we gone 10 years after the discovery? Yonsei Med J, 1998. 39(6): p. 520-5.

[34] Hall, B.G. and M. Barlow, Structure-based phylogenies of the serine beta-lactamases. J Mol Evol, 2003. 57(3): p. 255-60.

[35] Naas, T. and P. Nordmann, OXA-type beta-lactamases. Curr Pharm Des, 1999. 5(11): p. 865-79.

[36] Hall, B.G., S.J. Salipante, and M. Barlow, The metallo-betalactamases fall into two distinct phylogenetic groups. J Mol Evol, 2003. 57(3): p. 249-54.

[37] Walsh, T.R., et al., Metallo-beta-lactamases: the quiet before the storm? Clin Microbiol Rev, 2005. 18(2): p. 306-25.

[38] Payne, D.J., Metallo-beta-lactamases--a new therapeutic challenge. J Med Microbiol, 1993. 39(2): p. 93-9.

[39] Hudson, C.M., et al., Resistance Determinants and Mobile Genetic Elements of an NDM-1-Encoding Klebsiella pneumoniae Strain. PLoS One, 2014. 9(6): p. e99209. 